\title{
Analyzing Policymaking for Tuberculosis Control in Nigeria
}

\author{
Nura M. R. Ahmad $(D),{ }^{1,2}$ Cristina Montañola-Sales $\left(\mathbb{D},{ }^{2,3}\right.$ Clara Prats $(D),{ }^{1}$ Mustapha Musa, ${ }^{4}$ \\ Daniel López $\mathbb{1}{ }^{1}$, and Josep Casanovas-Garcia $\mathbb{}^{1,2}$ \\ ${ }^{1}$ Universitat Politècnica de Catalunya, Barcelona, Spain \\ ${ }^{2}$ Barcelona Supercomputing Center (BSC), Barcelona, Spain \\ ${ }^{3}$ IQS Barcelona, Ramon Llull University, Barcelona, Spain \\ ${ }^{4}$ Gombe State Primary Health Care Development Agency, Gombe, Nigeria
}

Correspondence should be addressed to Cristina Montañola-Sales; cristina.montanola@iqs.edu

Received 18 May 2018; Revised 25 September 2018; Accepted 11 October 2018; Published 7 November 2018

Guest Editor: Bernardo A. Furtado

Copyright (c) 2018 Nura M. R. Ahmad et al. This is an open access article distributed under the Creative Commons Attribution License, which permits unrestricted use, distribution, and reproduction in any medium, provided the original work is properly cited.

\begin{abstract}
Today, tuberculosis (TB) is still one of the major threats to humankind, being the first cause of death by an infectious disease worldwide. TB is a communicable chronic disease that every year affects 10 million people and kills almost 2 million people in the world. The main key factors fueling the disease are the progressive urbanization of the population and poverty-related socioeconomic factors. Moreover, the lack of effective tools for TB diagnosis, prevention, and treatment has decisively contributed to the lack of an effective model to predict TB spread. In Nigeria, the rapid urbanization along with unprecedented population growth is causing TB to be endemic. This paper proposes a mathematical model to evaluate TB burden in Nigeria by using data obtained from the local TB control program in the community. This research aims to point out effective strategies that could be used to effectively reduce TB burden and death due to TB in this country at different levels. The study shows that efforts should be oriented to more active case finding rather than increasing the treatment effectiveness only. It also reveals that the persistence of the disease is related to a large number of latently infected individuals and quantifies the lives that could be saved by increasing the notification rate using active case finding strategy. We conclude that undiagnosis is the bottleneck that needs to be overcome in addition to the incorporation, improvement, and/or strengthening of treatment management and other essential TB control measures in Nigeria.
\end{abstract}

\section{Introduction}

Tuberculosis (TB) is the most challenging infectious disease that humankind faces. It is estimated that in the last 200 years TB has killed one billion (1.000.000.000) people [1]. Currently, TB is still among the main worldwide causes of death by an infectious disease [2]. In 2017, there were 10.4 million new TB cases and 1.7 million related deaths worldwide [3]. It is a very silent disease that has eluded mankind for a very long time. It affects people of all social statuses, although most TB cases occur in resource-limited countries.

TB is caused by Mycobacterium tuberculosis (Mtb). This bacillus is transmitted by the inhalation of infected aerosols generated by active TB patients. The inhalation of the bacilli will usually lead to the trigger of an immune response that can have one of the three different clinical outcomes: (1) complete clearance of the pathogen, (2) latent TB infection (LTBI), or (3) progression to primary active disease $[4,5]$. LTBI occurs when the host's immune response manages the initial containment of the $M t b$ by developing and encapsulating granulomas. More often than not, the bacilli remain physically contained and immunologically constrained by these encapsulated granulomas throughout the lifetimes of the hosts $[6,7]$. During this process, an endogenous reinfection can produce new infection spotlights that will presumably undergo the same control dynamics [4]. However, even decades after infection especially in the case of immunocompromised hosts (like HIV patients), the control process can fail and the host can develop an active disease. On average, about $10 \%$ of the LTBI people develop active TB during their lives [8]. A latent infected host can be reinfected several times, thereby increasing the load of $M t b$ in its body and 
hence increasing the chance of progressing to active disease. According to the World Health Organization (WHO), there were an estimated number of 2 to 3 billion people with LTBI in 2015 [9], thus at risk of developing an active disease.

Overcrowding and time in contact with patients are key factors of TB infection. Paradoxically, TB is often seen as a XIX century disease, mainly associated with the poor living conditions of workers in crowded cities. Today, TB is reaching the highest number of cases in absolute numbers in the history [9] precisely because of the massive urbanization of populations. This fact is especially painful in Nigeria, a country that is undergoing rapid urbanization with a rapidly growing population. At the current growth rate of about $2.8 \%$ to $3.5 \%$ a year, it is estimated that Nigeria's urban population will double in the next two decades [10]. As a consequence, $\mathrm{TB}$ is endemic in Nigeria. The control of the disease is coordinated by the National Tuberculosis and Leprosy Control Program (NTBLCP), in line with the End TB Partnership initiatives whose ultimate target is to eliminate TB as a public health problem by the year 2050 (meaning reaching less than 1 case per million-person population) [11]. Despite current global control efforts to reduce TB, Nigeria's TB incidence is refusing to show any significant decline [9]. On March 14th, 2017, a wide circulated paper announced that Nigeria ranks 4 th in TB infection worldwide [12]. This was followed by the press release by the NTBLCP national coordinator. The statistics show that over $80 \%$ of TB cases in Nigeria are still undetected while the disease has been claiming millions of lives over the years in the country $[9,13]$.

Many studies show that more intensified case finding is needed especially in areas with higher prevalence of TB [1416]. For instance, Brewer et al. [17] indicated that active case finding of TB in homeless individuals in the USA is the most effective strategy that leads to a decrease in the death of the TB patients. Moreover, a study by Dodd et al. [18] indicates that policymakers need to alternate between active case finding and passive case detection strategy from period to period in order to have effective TB control. This paper aims to use mathematical modeling in order to evaluate the effect of an increase in diagnosis rate and treatment success on TB dynamics in Nigeria.

Assessing TB Policymaking: Mathematical Models for TB Transmission. Several mathematical models have been used to estimate long-term dynamics of TB to help to assess the development of strategies to control it. The literature on compartment models to describe complex systems is extensive [19-21]. Following a top-down approach, the population is divided into different compartments (e.g., susceptible, exposed, infected, and recovered in the case of an SEIR model) and specific fluxes are set between these compartments. The dynamics of the disease is therefore defined with ordinary nonlinear differential equations through rigorous mathematical analysis [22].

Many different studies have been able to draw important conclusions on TB dynamics by means of models and other methods [23-33]. Okuonghae and Ikhimwin [16], for example, developed a model which classified the population by their TB awareness level, a key factor which could affect the case detection rate. Another factor which could contribute to better adjust TB models is the HIV dynamics, especially in the sub-Saharan African region [34]. HIV patients have a higher risk of becoming infected and also of progressing to active disease once infected than non-HIV infected people. According to WHO [35], nearly all HIV-positive people with active TB will die.

By means of mathematical models, Wallis [36] was able to identify individuals with an innate resistance to $\mathrm{Mtb}$. He concluded that understanding the mechanisms of resistance may lead to therapeutic strategies to counter immune evasion by $M t b$. Moreover, models were also used by Wallis [36] to assess the affinity of LTBI reactivation when patients are administered with drugs with TNF blockers. Similarly, another research project by Moualeu-Ngangue et al. [37] worked with a model simulating the global TB dynamics. The study showed that TB spreading crucially depends on the basic reproduction number (number of new cases one case generates during its sick period, which is estimated to be between 10 and $15[36,38])$. The research was done in Cameroon where the roles of TB diagnosis, treatment, TB awareness level, and traditional medicine in the dynamics of TB were assessed. Song et al. [39] investigated the epidemiological time scales of TB and they evaluated the risk of infection from both close contacts (clusters) and casual contacts (random). They concluded that the risk of infection depends on the source of infection as well as on different environmental characteristics.

Lastly, Guzzeta et al. [40] presented three different ways to model TB dynamics: (1) an ODE model with no age structure and constant population size, (2) an agestructured, stochastic version of the ODE model, and (3) a sociodemographic Individual-Based Model (IBM). An IBM is a model formed by autonomous agents which interact among them, including their environment, to follow their objectives. The models were fitted to epidemiological data from Arkansas, USA. The authors concluded that different modeling techniques have their advantages and drawbacks and they should be chosen carefully. For example, an ODE model is best suited to describe the evolution of prevalence, incidence, and mortality. On the other hand, an IBM would be best to estimate the fraction of reactivated cases or to fit age-specific incidence of active TB.

Although there are many studies into TB epidemic, cause, spread, and suggestive measures for therapy and control, none of the aforementioned studies uses a mathematical model to evaluate the actual epidemic of TB in Nigeria using data obtained from the local TB control program in the community. This paper proposes a mathematical model that will be used to evaluate TB burden in this country. We aim to point out effective strategies that could be used to effectively reduce TB burden and death due to TB in Nigeria.

Specifically, we present a mathematical model that aims to investigate if more $\mathrm{TB}$ active case finding is needed in Nigeria, in order to help policymakers to make sound decisions in implementing effective TB control measures in the country. Section 2 shows the developed methodology and explains the proposed model. Sections 3 and 4 present and discuss the results obtained as well as their limitations and 
other possibilities for further research. Finally, the last section highlights the main conclusions drawn from this study.

\section{Materials and Methods}

Our goal is to develop a close-enough-to-reality mathematical model of the TB epidemic that can allow us to investigate effects of demographics and notification of TB epidemics in a population of Nigeria, as well as to estimate the actual burden of the disease including death toll and case fertility ratio in some of the regions in the country.

2.1. Model Description. Figure 1 represents the model diagram, which we briefly outline. Birth occurs at a constant rate $\Pi$ into the susceptible class $S$ with the assumption that all newborns are susceptible to $M t b . E_{1}, E_{2}, \ldots, E_{7}, E_{>7}$ represent the noninfectious population infected with tuberculosis without any clinical symptoms (LTBI) in their various years of infection; i.e., an individual who was infected recently (less than one year ago) will be assigned to $E_{1}$, while an individual who was infected last year would be assigned to the $E_{2}$ population. The last LTBI compartment, $E_{>7}$, consists of the whole latently infected population with more than 7 years of infection. $I_{1}$ and $I_{2}$ represent the population that is sick; i.e., they have an active disease with clinical symptoms and they can infect the general population; $T$ is the sick population that was diagnosed and is receiving effective chemoprophylaxis, thus unable to infect anyone. As shown in the diagram, only people in the $I_{2}$ compartment will be diagnosed and will move towards the $T$ compartment, while the $I_{1}$ compartment accounts for the missed TB cases. Death rates in the model depend on disease status; they are fixed into $\mu_{S}$ for susceptible population, $\nu_{1}$ for exposed classes, and $\mu_{I_{1}}, \mu_{I_{2}}$, and $\mu_{T}$ for sick individuals of $I_{1}, I_{2}$, and $T$, respectively. Based on the disparate time scale of natural death versus death due to TB disease, we assume that $\mu_{S} \leq \nu_{1} \leq \mu_{T} \leq \mu_{I_{2}} \leq \mu_{I_{1}}$.

Transmission of $M t b$ TB occurs following adequate contact between the sick infectious individuals $\left(I_{1}\right.$ and $\left.I_{2}\right)$ and the susceptible population. We assume that the latently infected $\left(E_{i}\right)$ are not infectious and thus not capable of transmitting the bacteria. We use the incidence expression $\alpha\left(\theta I_{1}+I_{2}\right) / N$ to indicate successful transmission of $M t b$ due to nonlinear contact dynamics in a large population [41]. $\alpha$ is the transmission rate that represents possible interactions that may occur among the susceptible population and the sick infectious population, which is defined as the average contact per unit time. Infectivity of $I_{1}$ is assumed to be lower $(0 \leq \theta \leq 1)$, as the undiagnosed long-term sick individuals probably reduce their activity due to their poor health conditions.

Newly infected individuals progress directly to the infected class $E_{1}$ and stay there for a period of one year, where the probability of developing active disease is $p_{1}$. If they do not become sick, they progress to $E_{2}$ (at a per capita rate $k_{1}$ ) the subsequent year, where the probability of developing the active disease is $p_{2}$. The progression through the latently infected compartment will continue yearly at a constant per capita rate $k_{1}$. Finally, all latently infected patients with more

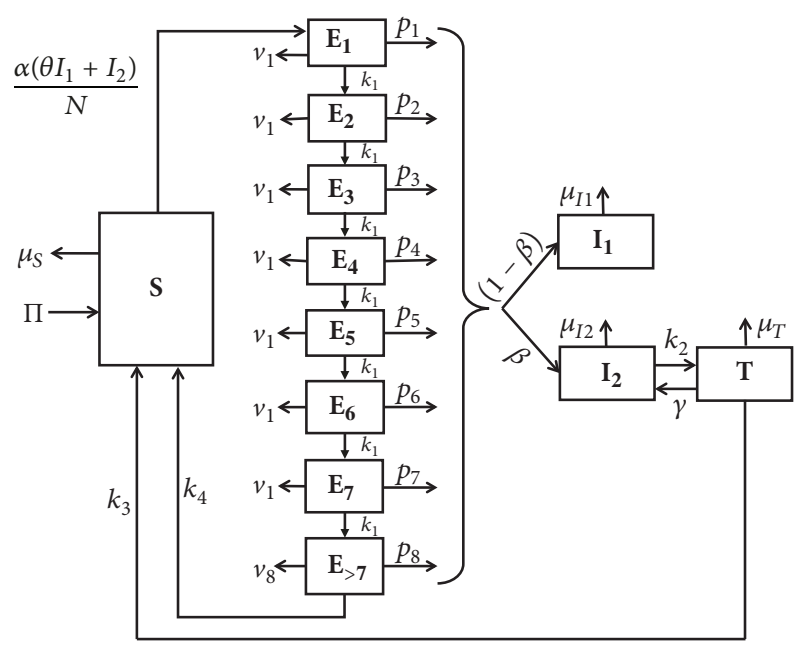

FIGURE 1: The model: a compartment model for tuberculosis transmission in Nigeria. The model shows the dynamic flow of tuberculosis (TB) including susceptible population $S$; the latently infected population are discerned based on the time since they were infected; therefore $E_{1}$ stands for people latently infected for a period of one year, $E_{2}$ stands for people infected for 2 years, etc.; $E_{>7}$ stands for people that have been infected for more than 7 years; two types of the nondiagnosed sick population are considered, $I_{1}$ and $I_{2}$, and the sick under treatment is shown as $T$. Birth occurs at constant rate $\Pi$. Transmission of $M t b$ depends on $\alpha\left(\theta I_{1}+I_{2}\right) / N$ and probability leading to active TB is given as $p_{i}=a i^{2}+b i+c$, where $i$ is the $i^{\text {th }}$ year of infection. $k_{1}$ indicate succession from one infected compartment to the next. A fraction of the sick, $\beta$, will be detected and notified; $k_{2}$ is related to diagnosis time delay before starting treatment and $\gamma$ indicates a fraction of relapse for the sick under treatment. Recovery rate is given as $k_{3}$ while $k_{4}$ represents the fraction of the infected population that gets rid of the disease spontaneously. We account for all courses of death, $\mu_{s}, v_{i}, \mu_{I_{2}}$, and $\mu_{T}$, and death due to active $\mathrm{TB}, \mu_{I_{1}}$.

than seven years of infection will remain in the compartment $E_{>7}$ for a very long period of time, where the probability of becoming sick $\left(p_{8}\right)$ is very low and later would be integrated back into the susceptible society at a constant per capita rate of $k_{4}$.

The probability of developing an active disease decreases across the latently infected compartment, $p_{i}$, which is assumed to be an autonomous decreasing quadratic function, given as $p_{i}=a i^{2}+b i+c$, where $i$ is the $i^{t h}$ year of infection. The sum $\left(\sum p_{i} E_{i}\right)$ represents the $10-13 \%$ of the latently infected population that will become sick in seven years or more, and $p_{1} E_{1}+p_{2} E_{2}$ is about $5-6 \%$ of the latently infected population that will become sick in the first two years of infection.

We define $T$ as the fraction of the sick infectious population that were notified and are receiving effective chemoprophylaxis, $k_{2}$ as the rate of effective per capita notification, and $k_{3}$ as the per capita rate of successful therapy completion. We assume that starting a treatment removes an individual from infectious class $I_{2}$ and places them into $T$. We also assume that individuals receiving chemoprophylaxis can abandon therapy, thus showing a relapse at a per capita rate $\gamma$. 
2.1.1. Model Formulation. We used a system of nonlinear differential equations to model the dynamics of individuals within the population settings. Setting $N(t)=S(t)+E_{1}(t)$ $+E_{2}(t)+E_{3}(t)+E_{4}(t)+E_{5}(t)+E_{6}(t)+E_{7}(t)+E_{>7}(t)+$ $I_{1}(t)+I_{2}(t)+T(t)$ and suppressing time dependence, $t$, for each variable, $N(t)$ represent the total population size at time $t$. The twelve model equations are

$$
\begin{aligned}
\frac{d S}{d t} & =\Pi+k_{4} E_{>7}+k_{3} T-\left(\frac{\alpha\left(\theta I_{1}+I_{2}\right)}{N}+\mu_{s}\right) S, \\
\frac{d E_{1}}{d t} & =\left(\frac{\alpha\left(\theta I_{1}+I_{2}\right)}{N}\right) S-\left(\nu_{1}+k_{1}\right) E_{1}+p_{1} E_{1}, \\
\frac{d E_{2}}{d t} & =k_{1} E_{1}-\left(\nu_{1}+k_{1}\right) E_{2}+p_{2} E_{2}, \\
\frac{d E_{3}}{d t} & =k_{1} E_{2}-\left(\nu_{1}+k_{1}\right) E_{3}+p_{3} E_{3}, \\
\frac{d E_{4}}{d t} & =k_{1} E_{3}-\left(\nu_{1}+k_{1}\right) E_{4}+p_{4} E_{4}, \\
\frac{d E_{5}}{d t} & =k_{1} E_{4}-\left(\nu_{1}+k_{1}\right) E_{5}+p_{5} E_{5}, \\
\frac{d E_{6}}{d t} & =k_{1} E_{5}-\left(\nu_{1}+k_{1}\right) E_{6}+p_{6} E_{6}, \\
\frac{d E_{7}}{d t} & =k_{1} E_{6}-\left(\nu_{1}+k_{1}\right) E_{7}+p_{7} E_{7}, \\
\frac{d E_{>7}}{d t} & =k_{1} E_{7}-\left(\nu_{1}+k_{1}\right) E_{>7}+p_{8} E_{>7}, \\
\frac{d I_{1}}{d t} & =\sum(1-\beta)\left(p_{i} E_{i}\right)+(1-\beta) p_{8} E_{>7}-\mu_{I_{1}} I_{1}, \\
\frac{d t}{d t} & =k_{2} I_{2}-\left(\gamma+k_{3}+\mu_{T}\right) T . \\
\frac{d}{d} E_{i}, 3 \ldots, 7 . & i=1,2,3 \ldots, 7 .
\end{aligned}
$$

Equation (1) describes the rate of change of the susceptible population $S$. There is a gain into this population through constant birth rate $\Pi$. A loss in this population occurs as a result of infection with $M t b$ with transmission rate $\alpha\left(\theta I_{1}+\right.$ $\left.I_{2}\right) S / N$ and constant death rate $\mu_{\mathrm{s}}$. Equations (2) through (9) represent the rate of change of the latently infected population over time. The rate of change of $E_{1}$ increases as a result of $M t b$ infection that results in latent infection at a rate $\alpha\left(\theta I_{1}+\right.$ $\left.I_{2}\right) S / N$ and decreases by developing active TB at a rate $p_{1} E_{1}$, natural death $v_{1}$, and movement to the second year of latent infection class at a rate $k_{1} E_{1} . E_{2}(t), E_{3}(t), \ldots, E_{>7}(t)$ increase by successive movement of latently infected individuals from one class to the next and decrease by progressive movement to the next infected class, natural death $\nu_{1}$, and developing of active TB at the rate $p_{i} E_{i}$ in each $i^{t} h$ latently infected class. A fraction $(1-\beta)$ of the $M t b$ infection which progress to active TB and were undetected decrease by death due to TB and other causes at the rate $\mu_{I 1}$, and the detected fraction are increased by relapse at a rate $\gamma T$ and decrease by diagnosis at a rate $k_{2} I_{2}$ and by death at a rate $\mu_{I 2}$. The rate of change of sick individuals under treatment is increased at a rate $k_{2} I_{2}$ and reduced at a rate $\gamma T$, recovery rate $k_{3} T$, and natural death at the rate $\mu_{T}$.

2.1.2. Model Calibration and Validation. In order to determine the effects of various parameters on the dynamics of TB in Nigeria, (1) to (12) are integrated by a Runge-Kutta method of order 4, using Matlab software (Ode45). We started by fitting our model to the 2000-2010 prevalence data on Nigeria from the official WHO reports and Nigerian epidemiological fact sheets [42]. The reason why this period was chosen is because of reliability of available data together with the fact that all parameters can be approximately considered constant for that period, which would not be applicable if the period was longer. We used the least squares curve fitting in Matlab, by specifying the lower and upper bounds of specific parameters to be estimated. The recruitment rate of the susceptible was chosen and calculated such that the population of the country remains constant during the simulation. The treatment efficacy $(1-\gamma)$ is considered to be $80 \%$ with probability of relapse taken as $20 \%$. The parameter values that gave the best fit are given in Table 1 and were obtained with $R^{2}=0.9992$.

The initial conditions were chosen in accord with available data when possible (i.e., for the total population, estimates of global LTBI population, the population in sick compartments, and the ratio $I_{1} / I_{2}$ ). The distribution of LTBI among time-since-infection compartments was also fitted in this process. We finally obtained these initial conditions (considering a population of 100,000):

$$
\begin{aligned}
& \left(S(0), E_{1}(0), E_{2}(0), E_{3}(0), E_{4}(0), E_{5}(0), E_{6}(0), E_{7}(0), E_{>7}(0), I_{1}(0), I_{2}(0), T(0)\right) \\
& \quad=(58303,6100,5000,4438,4020,3550,3140,2860,12250,284,55,0) .
\end{aligned}
$$

This corresponds to initial prevalence of 339 and a notification rate of $16 \%$. Figure 2 shows the epidemiological data together with the best fit of the model.

As a validation of the previous calibration, we took data from a wider period (1990-2015) and confronted it with the model. Keeping all the parameters constant but with a small change in the force of infection value, as well as in the initial conditions, the new $R^{2}$ was 0.9706 . Therefore, we assumed the calibration for the 2000-2010 period to be the baseline for our subsequent virtual experiments. 
TABLE 1: Summary description of parameters of the model fitted to Nigeria data (2000-2015).

\begin{tabular}{|c|c|c|c|}
\hline Parameter & Parameter description & Values $\left(\right.$ month $\left.^{-1}\right)$ & Source \\
\hline$\Pi$ & Birth rate in $S$ class & Constant & NA \\
\hline$\mu_{s}$ & Death rate in $S$ & 0.012 & calibrated \\
\hline$\mu_{i_{1}}$ & Death rate in $I_{1}$ & 0.146 & calibrated \\
\hline$\mu_{i_{2}}$ & Death rate in $I_{2}$ & 0.146 & calibrated \\
\hline$\mu_{T}$ & Death rate in $T$ & 0.01 & calibrated \\
\hline$v_{1}$ & Death rate in LTBI & 0.0108 & calibrated \\
\hline$\alpha$ & Contact rate of sick population & 3.54 & calibrated \\
\hline$k_{1}$ & Movement rate in LTBI population & $\frac{1}{12}$ & calibrated \\
\hline$p_{i}$ & Probability of developing active TB & $a i^{2}+b i+c$ & derived \\
\hline$\beta$ & Notification rate & 0.16 & [35] \\
\hline$\theta$ & Reduced probability for sick individuals in $I_{1}$ & 0.78 & calibrated \\
\hline$k_{3}$ & Recovery rate & $\frac{1}{6}$ & {$[35]$} \\
\hline$k_{4}$ & Inverse $\mathrm{TB}$ clearance rate & $\frac{1}{120}$ & calibrated \\
\hline$\gamma$ & Relapse rate & 0.20 & {$[35]$} \\
\hline$v_{8}$ & Death rate in $E_{>7}$ & 0.008 & calibrated \\
\hline$k_{2}$ & Inverse of diagnosis delay time & $\frac{1}{3}$ & [11] \\
\hline$a$ & Probability component for active TB & 0.001035 & calibrated \\
\hline$b$ & Probability component for active TB & -0.0152 & calibrated \\
\hline$c$ & Probability component for active TB & 0.06 & calibrated \\
\hline$(1-\gamma)$ & Effective TB treatment rate & 0.80 & [35] \\
\hline
\end{tabular}

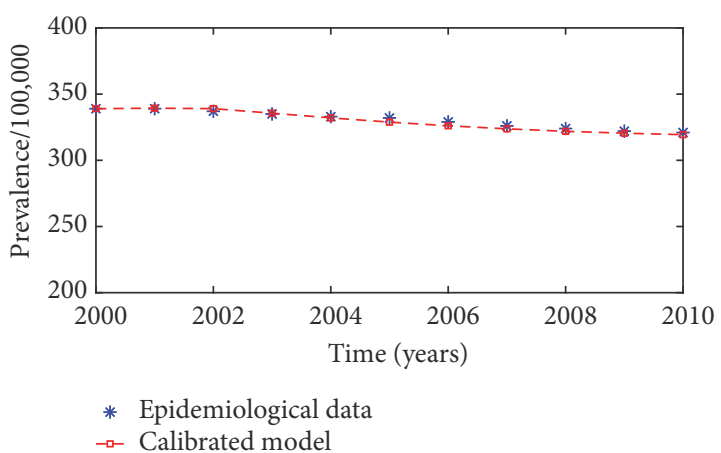

FIGURE 2: Model simulation calibrated against tuberculosis prevalence data in Nigeria: The figure shows the tuberculosis prevalence curve of the model, calibrated with 2000-2010 data, compared with the data estimates from WHO in that period. The model prevalence curve is obtained by solving (1) to (12), using the initial conditions in (13) and parameter values in Table 1 . The goodness-of-fit measure $\left(R^{2}\right)$ for simulation trajectories was evaluated and found to be 0.9992 .

2.2. Facts and Hypotheses. For the purpose of this paper, we assume a constant birth rate $\Pi$. All newborns into the model susceptible class are uninfected, and TB/HIV coinfection is not explicitly modeled. These assumptions are simplifications that are not relevant to the current purpose, which is the identification of significant damage caused by poor case detection in the TB control program. They will be modified in a subsequent study in order to account for the effects of changes in the birth rate over time and to evaluate the significance of TB/HIV coinfection.

We propose to use the model in the exploration of different situations in Nigeria as a virtual experiments platform. The situations evaluated have been selected to start working on four wide hypotheses, although the final confirmation of all of them would require further research including onfield projects and incorporating experts from other involved disciplines. The four starting hypotheses are the following:

(1) TB prevalence still remains a major health challenge in Nigeria due to poor case detection

(2) Increase in effective treatment may not necessarily cause a significant decrease in the prevalence of TB in the country if active case finding is not implemented. In other words, increasing the notification rate is necessary for decreasing TB transmission in Nigeria

(3) The persistence and nondecreasing dynamics of TB in Nigeria are also related to a large number of latently infected individuals

(4) Poor notification rate has resulted in a large number (hundreds of thousands) of deaths of TB patients

\section{Results}

Numerical simulations of the model allowed us to estimate some important parameters associated with TB in Nigeria. In addition, we were able to observe and quantify the effect 
of the infected population on the prevalence of TB in the country. They also allowed us to make a distinct connection between the notification parameter and the death toll on the sick population (infectious) population, the relationship between the death and the prevalence of the disease in the country. To illustrate these effects, we divide this section into 3 subsections. Hypotheses 1 and 2 were used to explain the case detection and how effective treatment affects TB dynamics in Nigeria; we made a prediction of new TB cases and provide an alternative strategy for TB control. Hypothesis 3 was used to explain the relationship between LTBI and the dynamics of TB prevalence. Finally, by means of Hypothesis 4 we quantified the death due to TB and estimate the death toll due to TB for the next decade in Nigeria, and we also estimate lives that could be saved with the alternative strategy proposed.

\subsection{Hypotheses 1 and 2: Case Detection and Effective Treat-} ment. The model was successfully fitted into WHO data of TB prevalence in Nigeria. Then, we compared the fitted tendency with predictions regarding an increase in notification parameter. The parameter values obtained after fitting the model into data of Nigeria are briefly summarized and discussed in Table 1. As shown in Figure 2, the obtained baseline simulation represents the epidemic TB situation in Nigeria successfully. Values for many parameters were determined from vital statistics: TB data from NTBLCP in Gombe State, official TB data from the World Health Organization (WHO), and other recent literatures mentioned in Table 1. When the values could not be estimated from data or literature, as in the case of the parameters associated with the contact rate, they were obtained with the model's fitting into epidemiological data $\left(R^{2}=0.9992\right)$.

Recall, WHO estimates that about $30 \%$ of the world population is infected with LTBI [43]. However, the model revealed that about $34-37 \%$ (depending on the choice of contact rate for the sick population and some other parameters that where calibrated) of Nigeria's population is latently infected with TB. This population is distributed across the various infected classes ranging from $E_{1}$ to $E_{>7}$, and the time since infection in this population was also fitted.

We then used our model to investigate how the notification of new TB cases affects the dynamics of TB in Nigeria. We tested the hypothesis that the nonimprovement in notification observed in the situation of Nigeria may partially explain why the prevalence of $\mathrm{TB}$ in this country is very persistent and not declining as compared to others, showing an incidence rate between 400 and 500/100,000 (more than 130 times the incidence rate in the USA) [4446]. The notification rate of TB in Nigeria is about $16 \%$ [35]. Therefore, initial conditions for new TB cases were calculated by assigning $16 \%$ of new cases to $I_{2}$ (i.e., the compartment that gathers the sick population that goes to hospital and receives care at some point), and $84 \%$ were assigned to $I_{1}$ (i.e., the population that remain sick throughout their life cycle until death).

Figure 3 shows the fitting of the model into the prevalence of TB in Nigeria, namely, baseline, together with some

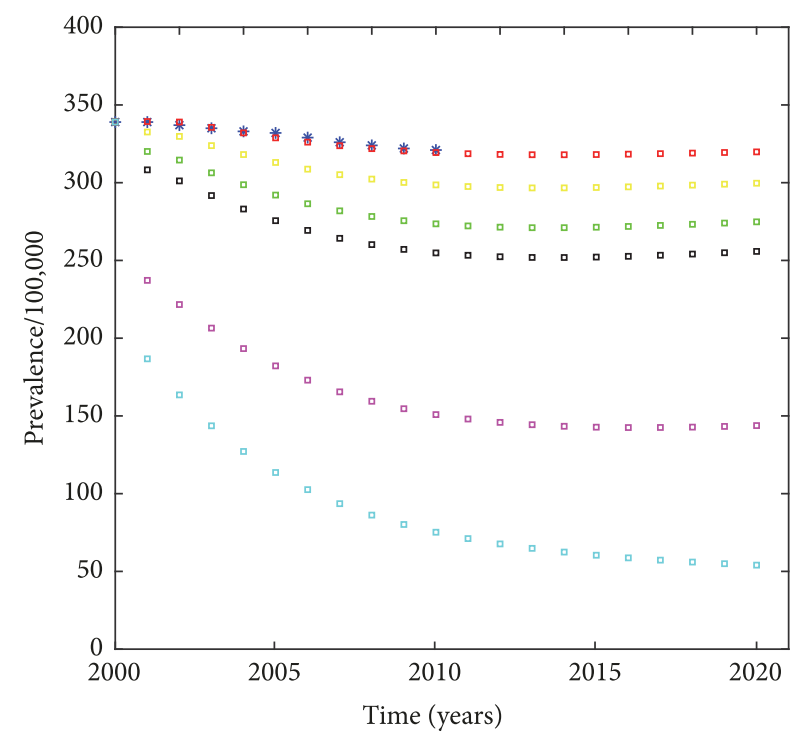

$$
\begin{array}{ll}
\text { * Epidemiological data } & \square 30 \% \text { Notification } \\
-16 \% \text { Notification } & \square 60 \% \text { Notification } \\
-20 \% \text { Notification } & \square 85 \% \text { Notification } \\
-25 \% \text { Notification } &
\end{array}
$$

FIGURE 3: Hypothesis 1: baseline simulation and effect of the notification rate. Model fitted to the prevalence of tuberculosis in Nigeria. The blue star represents the epidemiological data of tuberculosis prevalence while the red line-circle represents the model simulation. The fitting was done with $16 \%$ of the sick population being notified. The experiment was repeated with 20\% (yellow curve), $25 \%$ (green curve), 30\% (black curve), 60\% (magenta curve), and 85\% (cyan curve) notification, respectively.

simulations where an increase in the notification rate was explored. The mean prevalence for the epidemiological data is 330.64 with a standard deviation of 6.7 , while the mean for the model is 330.39 with a standard deviation of 6.6 and an $R^{2}$ coefficient of 0.9992 (Table 2). The model is pretty consistent with the epidemiological data. The mean resultant prevalence for each simulated notification rate is recorded in Table 2 as well. These results show a clear decrease in the prevalence of TB when the notification of the new TB cases increases.

In the baseline simulation, the model predicted 62,000 deaths due to TB in the year 1990, 72,000 deaths in the year 1995 , and 118,000 in the year 2014. These results are within the range of annual TB deaths estimated by WHO [35]. Figure 4 shows the predicted decline in prevalence after 10 years for each of the notification rates tested. In fact, a simply $10 \%$ relative increase in case notification of people with active $\mathrm{TB}$ reduces the TB prevalence by $15 \%$ when compared with the current situation. Predicted TB deaths also would decline by more than $15 \%$ when compared with the baseline simulation that fitted the epidemiological data.

A new simulation series was designed in order to explore the effect of an improvement of effective treatment, i.e., a decrease in $\gamma$. The results showed that a $10 \%$ improvement of effective treatment among active TB individuals produces a decline of only $5 \%$ in the prevalence of TB and related deaths compared with the baseline fitting, as shown in Figure 5(a). 
TABLE 2: Summary of the mean prevalence of data and model simulations, as well as $R^{2}$ of the fitting.

\begin{tabular}{lccc}
\hline Case detection rate & Mean prevalence & Standard deviation & References \\
\hline Epidemiological data & 330.64 & 6.7 & {$[35]$} \\
Model 16\% Notification & 330.40 & $6.8, R^{2}=0.9992$ & Model \\
Model 20\% Notification & 311 & NA & Model \\
Model 25\% Notification & 288 & NA & Model \\
Model 30\% Notification & 266 & NA & Model \\
Model 60\% Notification & 156 & NA & Model \\
Model 85\% Notification & 105 & NA & Model \\
\hline
\end{tabular}

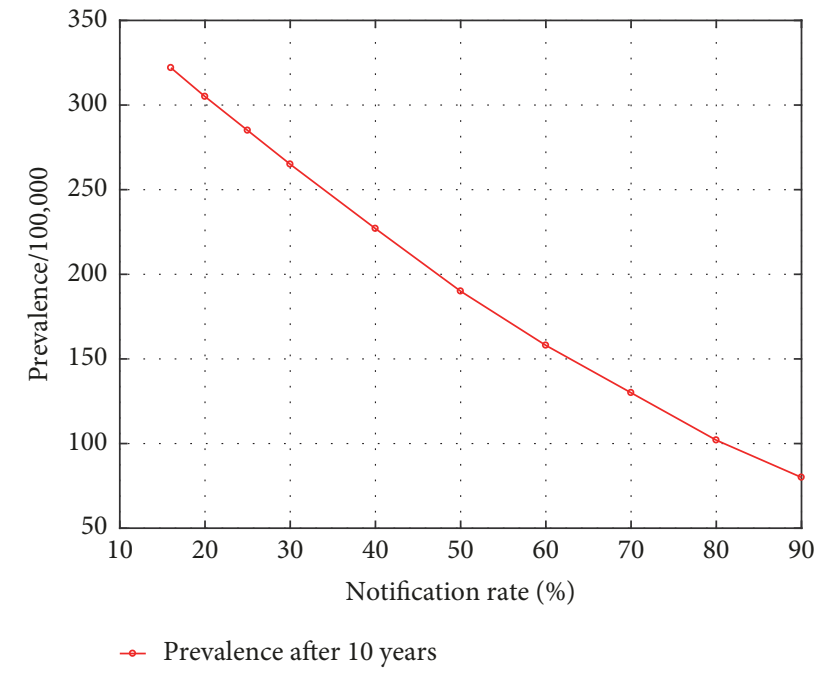

FIgURE 4: Hypothesis 1: evolution of tuberculosis prevalence in Nigeria after 10 years of simulation with different notification rate. Each point on the curve shows the prevalence of tuberculosis in Nigeria when simulated with the corresponding notification value on the $\mathrm{x}$-axis for 10 years.

Given these results, the most effective intervention for reducing future TB cases and related deaths in Nigeria as predicted by the model is active case finding.

A final virtual experiment was designed in order to explore the effect of an improvement of effective treatment in a context with a high case detection rate. In this simulation series, the detection rate was fixed at $80 \%$ and different treatment success rates were explored. The results showed that, in this case, the improvement in treatment effectiveness would provide a significant decrease in $\mathrm{TB}$ prevalence (Figure 5(b)). With a notification rate of $80 \%$, the increase in effective treatment by $10 \%$ will lead to a decline in the prevalence of $23 \%$ and associated deaths by almost $30 \%$. In the case of a poor case detection rate, increase in $10 \%$ of effective treatment is associated with only $5 \%$ decrease in prevalence and related deaths. Therefore, an increase in the treatment effectiveness is only relevant if it is combined with active case finding or in a scenario where the notification rate is significantly high. Nevertheless, with the growing problem multidrug-resistant $\mathrm{TB}$, investment in treatment becomes more relevant and needs to be maintained.
3.1.1. TB Prediction in Nigeria. Looking at the current TB situation in Nigeria, one can wonder what the situation will be if all parameters are left unchanged especially in terms of passive case finding. We envisioned this scenario and with the help of the baseline simulation results, the following results are presented. Figure 6(a) shows the predicted evolution of TB when the notification rate is gradually increased, while Figure 6(b) shows the effect of a sudden increase in this parameter. The simulation starts with the baseline that represents the current situation (16\% notification, red-dashed line). Keeping the notification at this rate without any changes may keep the prevalence of TB in the country between 350 and 320/100,000 even after 40 years from the present day. This is precisely the present situation of TB in Nigeria, where there have not been any significant changes or decline in TB prevalence for the past 25 years $(323 / 100,000$ in 1990 and $332 / 100,000$ in 2015 [35]). Increasing the notification rate up to $20 \%$ in 10 years could keep the prevalence between 300 and 290/100,000 people. If no more effort is made to increase the notification further, the prevalence would remain in this range even after a period of 50 years. Increasing the notification further to $25 \%$ in another 10 years would reduce the prevalence to between 290 and 260/100,000 people, and a further increase in notification to $30 \%$ would bring down the prevalence to between 220 and 240/100,000. We repeated the experiment with a sudden increase in the notification from $16 \%$ to $30 \%$ as seen in Figure 6(b) when the prevalence would remain in the range of 270/100,000 to 280/100,000.

For a successful and reasonable decline in TB cases in Nigeria to be achieved, case finding needs to be active and increased from period of time to time. Although a 30\% notification rate is viable, a real $\mathrm{TB}$ prevalence decrease in Nigeria can only be achieved with a notification rate around 80\%. Both strategies presented in Figures 3 and 6 produce the same results in the long run in terms of TB prevalence decline. However, in terms of public health, a progressive decreasing strategy would be more feasible due to the economic resources that need to be allocated by health authorities. In contrast, increasing the notification rate by a large percentage could be very ambitious and resource demanding. At this junction, the most important point is to be able to make interventions as soon as possible given the long time required to actually see changes in TB prevalence.

3.2. Hypothesis 3: Latently Infected Population. Although it is known that latently infected individuals are connected 


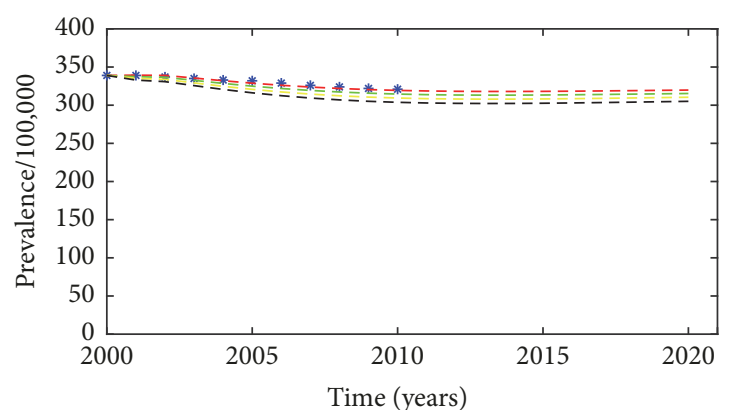

* Epidemiological data

- - $80 \%$ Treatment success rate 85\% Treatment success rate 90\% Treatment success rate - _ 95\% Treatment success rate

(a) $16 \%$ case notification

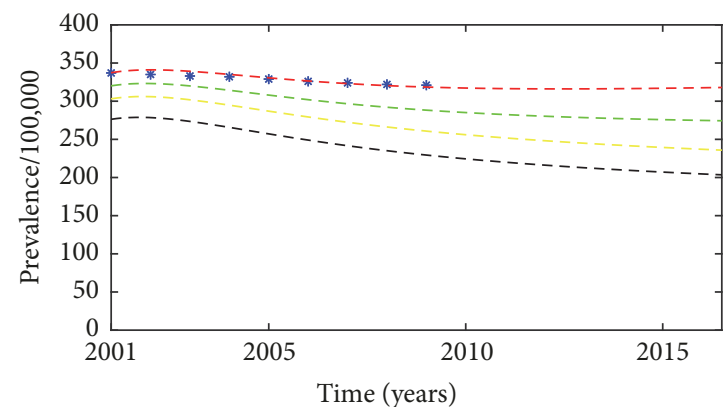

* Epidemiological data

- - $80 \%$ Treatment success rate 85\% Treatment success rate 90\% Treatment success rate - - 95\% Treatment success rate

(b) $80 \%$ case notification

FIGURE 5: Hypothesis 2: effective treatment in the population with good surveillance and poor surveillance. (a) The figure depicts a relative increase in effective treatment from $80 \%, 85 \%$, and $90 \%$ to $95 \%$ in a population with poor case detection, where the notification rate is only $16 \%$. (b) The figure shows a significant decrease in prevalence due to increase in effective treatment from $80 \%, 85 \%$, and $90 \%$ to $95 \%$, in a population with very good case detection, where the notification rate is $80 \%$.

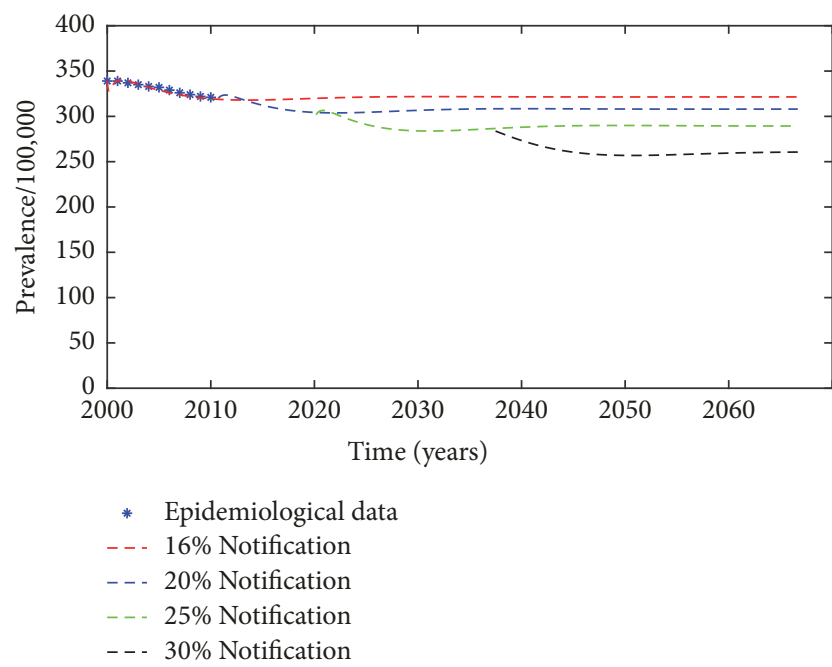

(a)

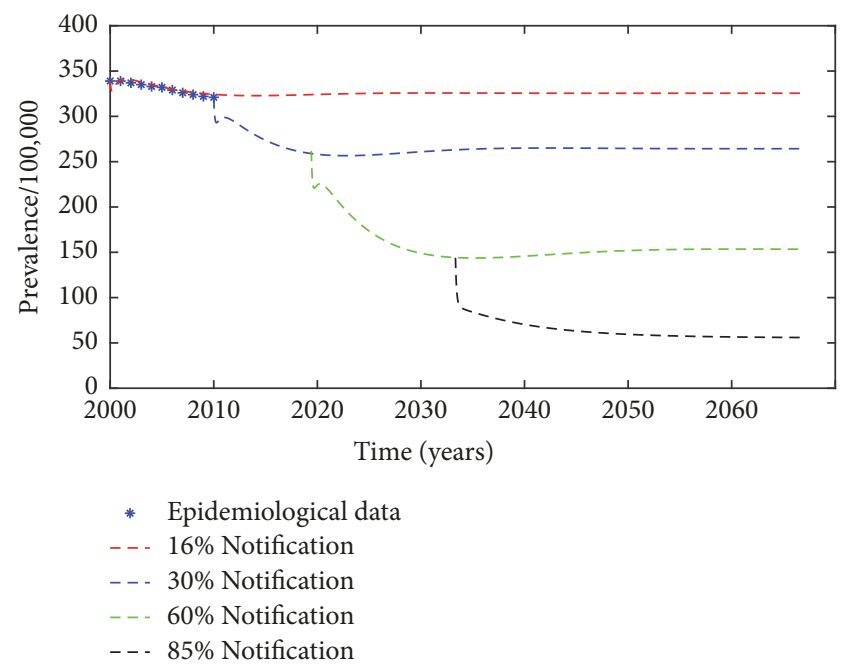

(b)

FiguRE 6: Hypothesis 1: effective strategies in a gradual increase in tuberculosis notification in the case of poor tuberculosis surveillance. (a) Result of the prediction made by the model when the notification of new tuberculosis cases is gradually increased. The red curve shows the poor notification rate situation, which represents the present situation of tuberculosis in Nigeria (the blue stars). The rest of the curves represent a change in case detection and increasing in the notification of new cases by some percentages over a period of time. (b) Result of the prediction made when the notification of new tuberculosis cases is suddenly increased.

with the incidence of $\mathrm{TB}$, it is not completely clear how this connection can influence the dynamics of the disease in a population. Our first hypothesis implied that poor case detection leads to a higher number of unnotified TB cases, which leads to a high number of latently infected individuals that are at risk of developing active TB, thus resulting in persistent high TB prevalence. Our model was also used as a virtual experimental device to test the effect of the latently infected population in Nigeria, which can provide significant information that can be very difficult or impossible to guess otherwise. We are going to show why the prevalence of TB in Nigeria is substantially high and nondecreasing for a very long period of time.
We explored the effect of increasing the notification rate or the treatment success on the LTBI population (Figure 7). Figure 7 (a) shows the result of various simulations of the latently infected population carried out with different notification parameters. The latently infected population consistently declines with each increase in notification rate. This result explains why a population with a poor case detection program can fail to achieve significant progress in reducing new $\mathrm{TB}$ cases, because the latently infected population remains the same for a very long period of time due to what is called the replacement principle, where each sick TB patient produces at least one sick patient before death or progress to receiving treatment (the number of new cases 


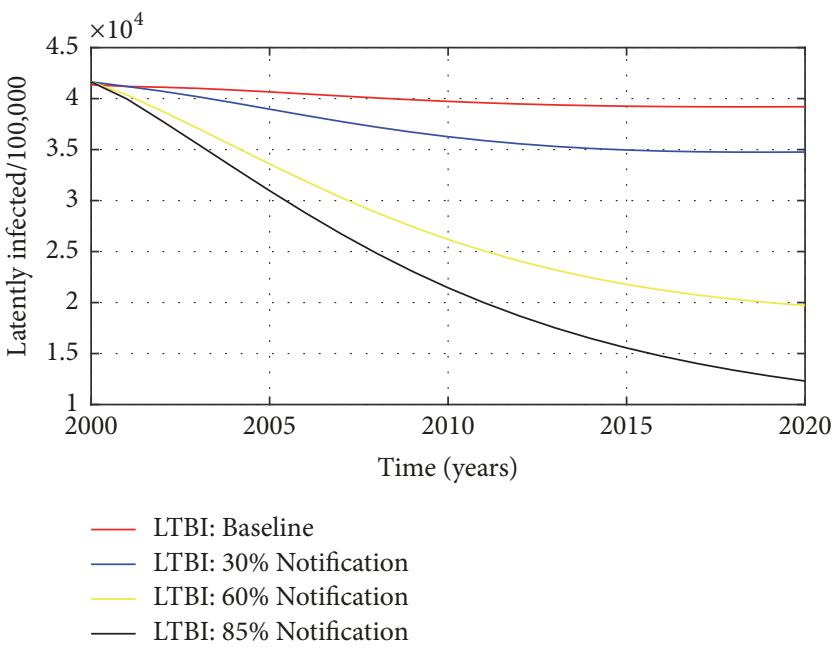

(a)

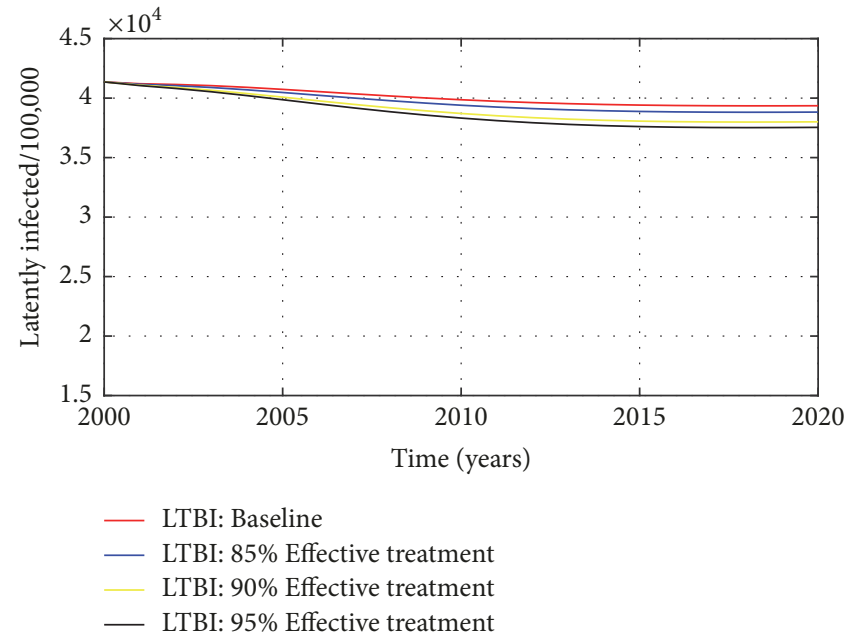

(b)

FIGURE 7: Hypothesis 3: changes in latent tuberculosis with respect to an increase in notification rate or in effective treatment. (a) Simulated dynamics of the latently infected population (LTBI) with different notification levels, with all other parameters kept constant in the population of Nigeria. The red curve shows the LTBI population that remained constant for more than 20 years, thereby producing new sick people constantly. With an increase in notification up to 30\%, the pool of the LTBI starts decreasing (blue curve), and a further increase in notification shows a much higher decrease in the pool of LTBI (black curve, 85\% notification rate). (b) Simulation of the LTBI population with improvement in effective treatment, with all other parameters kept constant in the population of Nigeria. There is little or no change in the number of LTBI cases before and after increasing the effective treatment.

that receive treatment is very low). This type of situation guaranteed the consistent production of newer TB cases if active case finding is not implemented.

The increase in notification rate affects not only the active new TB cases but also the latently infected population. A $10 \%$ increase in notification was associated with a $38 \%$ decline in latent infected TB (Figures 7(a) and 8), while a 10\% increase in effective treatment showed only a $2.9 \%$ decline in the latently infected population (Figure 7(b)).

\subsection{Hypothesis 4: Poor Notification and Mortality in Sick Pop-} ulation. We finally tested the hypothesis that low notification rate has resulted in massive deaths in the sick population. This phenomenon might be a mystery in Nigeria as not every death is reported to the authorities. Only the deaths that occur in a notified case of $\mathrm{TB}$ are recorded as a death due to this disease. As the notification rate of TB in Nigeria is just $16 \%$, one can wonder how many death cases due to $\mathrm{TB}$ went unnoticed. As mentioned earlier, we used the notification of $16 \%$ to fit the data to the model.

Figure 9 shows the estimated TB-related mortality per a hundred thousand individuals in the population of Nigeria obtained from the model. The present-day mortality rate in Nigeria is shown by the red curve, which shows that mortality due to TB in Nigeria is between 70 and 65 individuals per 100,000 individuals. This result is very consistent with the report from WHO [35]. The model shows that an increase in the notification by $10 \%$ would lead to the decrease in mortality by $20 \%$. Actually, we see from Figure 9 that if the notification is increased from $16 \%$ to $25 \%$, the mortality declines to less than $60 / 100,000$ individuals, which implies hundreds of thousands of lives that would be saved. In

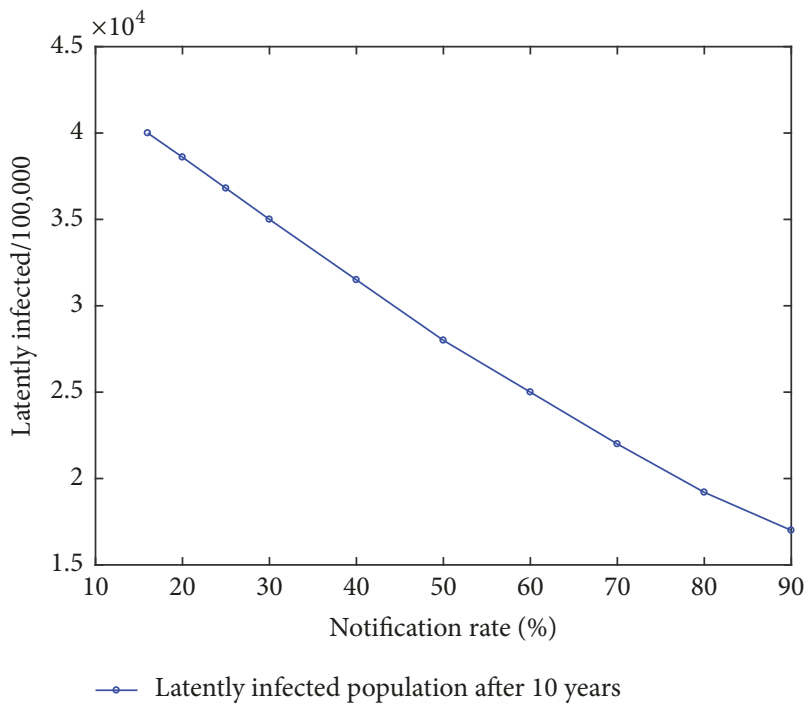

FIGURE 8: Hypothesis 3: latently infected population after 10 years of simulation of the model with different notification levels. Each point of the curve represents the corresponding population of the latently infected compartments, when the model is simulated with the corresponding notification rate on the $\mathrm{x}$-axis.

order to better understand these dynamics, we estimated the mortality rate in Nigeria after 10 years of simulation with the model by using different notification parameters, starting from the current situation in Nigeria (notification rate of 16\%). The result of this experiment is shown in Figure 10.

The expected cumulative number of deaths due to $\mathrm{TB}$ with the $16 \%$ notification after 10 years of simulation is 


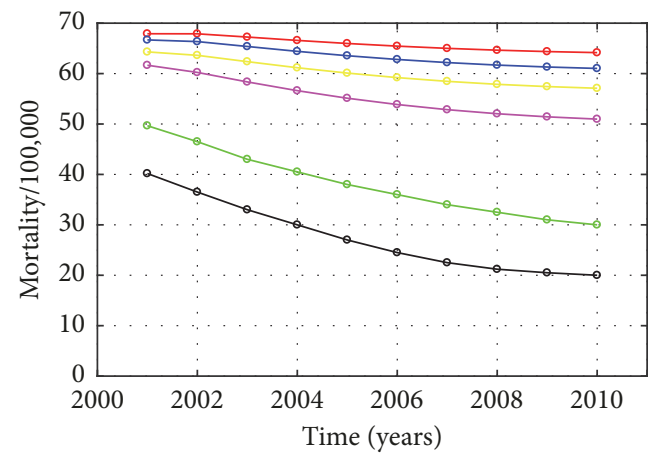

$\rightarrow 16 \%$ Notification
$\rightarrow \quad 20 \%$ Notification
$\rightarrow \quad 25 \%$ Notification
$\rightarrow 30 \%$ Notification
$\rightarrow 60 \%$ Notification
$\rightarrow \quad 85 \%$ Notification

FIgURE 9: Hypothesis 4: The figure shows the decrease in mortality due to tuberculosis in the sick population for a period of 10 years with different notification levels. The red curve represents the current mortality due to tuberculosis in Nigeria (baseline), and the rest of the curves show what the situation would look like if the notification were to be different.

$4,748,971$. An increase in notification to a rate of $20 \%$ could save up to 292,244 lives within this period. If the notification rate was increased to $25 \%$, up to 584,489 lives could be saved within this period.

Although the estimated TB prevalence in Nigeria from 1990 to the year 2016 remains the same, the estimated number of new cases increases annually. These are attributed to the increase in the population of the country, but, at the same time, it shows a massive increase in the number of deaths due to TB in the population as estimated by the model. The estimated new TB cases rise from 310,000 in 1990 to more than 600,000 in 2015, and the estimated number of TB-related deaths by the model rises from 62,000 in 1990 to more than 120,000 in 2015 . If the population of Nigeria continues to grow at the rate of $3.2 \%$ annually [47] and the case finding remains passive at $16 \%$ notification, then TB-related deaths will reach up to $3,456,640$ in the year 2030; this is twice the current number of deaths due to TB in the world reported by WHO in 2017 [3]. Depending on availability of funds, political willingness to implement active case finding in Nigeria, and the strategy implemented, millions of lives could be saved from 2018 to 2030. Globally, the model predicted a total death number of 11,872,530 individuals from 2017 to 2030. An increase in case detection by $15 \%$ within this period could save up to $2,557,160$ lives and would reduce the incidence by more than $30 \%$.

\section{Discussion}

Epidemiological data from Nigeria shows that the TB situation in this country is very persistent over time, in spite of the efforts made by all the stakeholders battling the disease.

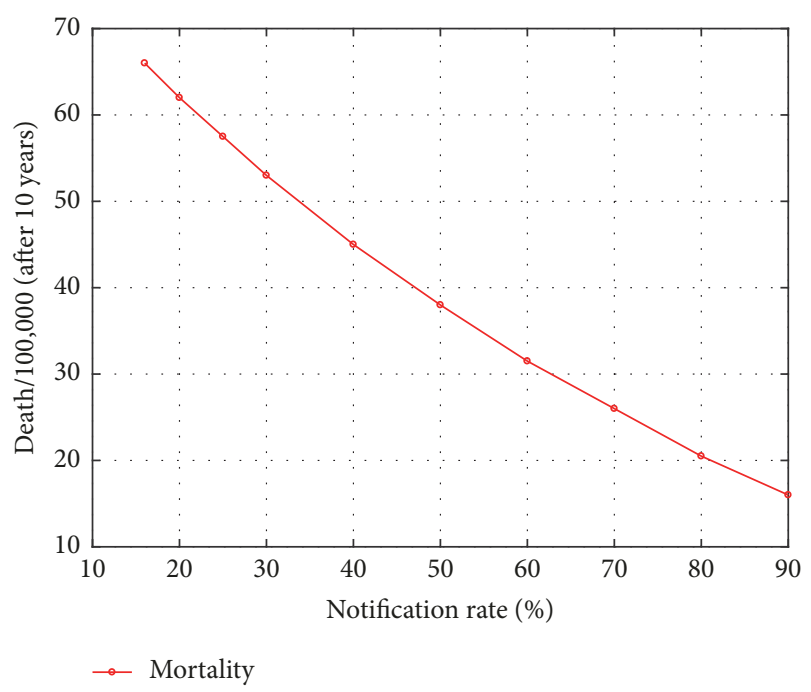

FIGURE 10: Hypothesis 4: the result of 10-year simulation of the model using different notification rates. Each point on the curve represents the mortality in the sick population after the model is simulated for 10 years with the corresponding notification rate on the $\mathrm{x}$-axis.

While TB prevalence in Nigeria in 1990 was 323/100,000 individuals, today it is around the same number $(330 / 100,000)$ according to $\mathrm{WHO}$. The same situation is observed in the number of deaths due to the disease $(70 / 100,000$ today compared to $65 / 100,000$ in 1990 ). The reason for this steady state of the TB epidemic in the country is still unclear. Many associate it with poverty and population growth. Nigeria recently overtook India as the world's poverty capital [48] with about 87 million people in abject poverty [48]. Its unprecedented demographic growth (from 95,62 million people in 1990 to 206,20 in 2018) is mainly due to a very high fertility rate (5.526 according to the World Bank in 2016). Others presume that there is a need for a better strategy in order to fight the disease towards a significant decline in the near future [11].

Nigeria is a particular setting where several healthcare options (medical pluralism) including orthodox medicine (public, private, or drugstores), traditional medicine, and spiritual healers operate freely [11]. The public health facilities where the TB control program operates are distanced from the citizens. More often than not, these health facilities are not the first choice during health seeking decisions. Unlike the theory of Dim and Dim [11] and Zwerling et al. [49], we believe that high TB prevalence in Nigeria has a lot to do with policies of its TB control program, as stated in other studies [18, 45, 50,51]. Today, Nigeria is ranked as number 3 in the world in terms of TB burden [52] just behind China and India. The country has adopted passive case finding as recommended by WHO [42], based on a study conducted in India $[53,54]$. Nevertheless, the Indian context has completely different demographic, cultural, and literacy settings from Nigeria. However, successful TB control only happens when social and cultural factors are taken into consideration [55]. It is therefore imperative to revise and consider the means of extending the NTBLCP strategy. 
In this study, we applied epidemic models to real populations to draw conclusions about one of the leading health challenges in Nigeria. We numerically showed that an increase in the diagnosis rate together with a treatment's success of TB patients is an essential step towards TB control in Nigeria. In order to increase notification rate of TB in Nigeria using the current passive case detection strategy, the people at the community level should be empowered with adequate knowledge of the growing burden of the disease and accessible potentials for a cure. Alternatively, active case finding strategies could be explored and implemented, although the existence of many remote communities in some Nigerian areas would dramatically make this task difficult. In any case, the analysis reveals that, with current strategies, it will be impossible to actualize the aims of the End TB strategy, with targets to reduce TB deaths by $95 \%$ and to cut new TB cases by $90 \%$ between 2015 and 2035 [56].

Even with a high increase in the notification and without considering HIV/TB coinfection and the problem of multidrug-resistant (MDR) strains, mortality from TB in Nigeria will still be dramatic for the next few decades to come. Two main facts will contribute to this increase in mortality: first, Nigeria's population growth of more than $3.2 \%$ annually $[47,57]$ and, second, the resulting deaths due to TB which are expected to drastically increase by 2030 unless effective TB control is implemented in the country. The results we obtained in our study confirm this situation. This issue poses a great threat not only to Nigeria but also to the entire world's TB program and to the ambitious End TB Partnership [56] by 2030.

The increase of TB diagnosis by applying active case finding along with specific actions to guarantee TB treatment's adherence and success could be used to achieve the goals set by WHO in Nigeria by reducing TB mortality significantly, TB prevalence, and LTBI population. Perhaps the time has come for a critical reexamination of the costs, risks, and benefits of active case finding in this country. Indeed, the strategies evaluated in this paper would require substantial resources and extraordinary mobilization of international attention, since the greater part of $\mathrm{TB}$ control program funding comes from external sources [42]. We have not attempted to estimate the cost difference of the control strategies proposed. Initiating and sustaining such a largescale effort of active case finding would be very challenging. However, the first step needs to include both a realistic assessment of implementing these ambitious initiatives and the identification of an optimal way to implement them. For that, the socioeconomic context of the different Nigerian territories and the social inequalities within and between them should be taken into account, so that they can be transformed into feasible policy recommendations. Furthermore, success could not be guaranteed without both realistic evaluation of the costs of pursuing active case finding and the treatment of new diagnosed TB sick individuals.

Our results exploring different scenarios show the high relevance of increasing the notification rate in contrast to the modest effects of an improvement of treatment efficacy only. Nevertheless, the treatment efficacy would probably turn out to be crucial if we included the problem of MDR, which would be addressed in further studies. Despite contemplating the absence of MDR as a limitation of the model, our conclusions cannot be questioned, since the main problem is still the pool of nondiagnosed TB sick individuals. Future studies could also include the study of the reinfection effect in the model. This new setting would allow a detailed study of the LTBI population and its distribution between old and recent infections. Nonetheless, the reinfection effect would not change the main dynamics given by the current model and the recommendations that arose from it.

\section{Conclusions}

The model shows that WHO targets stated by the End TB strategy [56] would not be achieved with the current public management of TB in Nigeria. In fact, its forecasted situation for 2030 suggests being far away from ending the TB epidemics as aimed in the United Nations Sustainable Development Goals under Goal 3. The high percentage of undiagnosed TB sick individuals in this country is a huge obstacle for the target incidence's disease. It is essential to increase the number of the diagnosed and successfully treated, thus requiring not only a significant economic effort but also a boost in social work in the community. This result is valid not only for Nigeria, but also for such countries where nondiagnosis is a serious problem. In all cases, a significant TB incidence's decrease can only be achieved by both increasing the notification rate to the $80 \%-90 \%$ range and providing appropriate management of diagnosed patients. With both measures, new infections can be prevented and the treatment's success can be guaranteed. This situation is the bottleneck that needs to be overcome in addition to the incorporation, improvement, and/or strengthening of other essential TB control measures such as directly observed treatment or contact tracing.

\section{Data Availability}

The epidemiological data supporting this study are from previously reported studies and datasets, which have been cited. The processed data are available from the corresponding author upon request.

\section{Conflicts of Interest}

The authors declare that they have no conflicts of interest.

\section{References}

[1] T. Paulson, “A mortal foe," Nature, vol. 502, pp. S2-S3, 2013.

[2] R. M. Houben, P. J. Dodd, and J. Z. Metcalfe, "The Global Burden of Latent Tuberculosis Infection: A Re-estimation Using Mathematical Modelling," PLoS Medicine, vol. 13, no. 10, p. e1002152, 2016.

[3] World Health Organization, “Global Tuberculosis Report," 2017.

[4] P.-J. Cardona, "Patogénesis de la tuberculosis y otras micobacteriosis," Formación médica continuada: infecciones por micobacterias, vol. 36, no. 1, pp. 38-46, 2018. 
[5] K. Bhatt and P. Salgame, "Host innate immune response to Mycobacterium tuberculosis," Journal of Clinical Immunology, vol. 27, no. 4, pp. 347-362, 2007.

[6] P. Peyron, J. Vaubourgeix, Y. Poquet et al., "Foamy macrophages from tuberculous patients' granulomas constitute a nutrientrich reservoir for M. tuberculosis persistence," PLoS Pathogens, vol. 4, no. 11, 2008.

[7] R. L. Hunter, "Pathology of post primary tuberculosis of the lung: an illustrated critical review," Tuberculosis, vol. 91, no. 6, pp. 497-509, 2011.

[8] W. W. Stead, J. P. Lofgren, E. Warren, and C. Thomas, "Tuberculosis as an Endemic and Nosocomial Infection among the Elderly in Nursing Homes," The New England Journal of Medicine, vol. 312, no. 23, pp. 1483-1487, 1985.

[9] World Health Organization and Others, "Global tuberculosis report 2016," 2016.

[10] A. Aliyu and L. Amadu, "Urbanization, cities, and health: The challenges to Nigeria - A review," Annals of African Medicine, vol. 16, no. 4, pp. 149-158, 2017.

[11] C. Dim and N. Dim, "Trends of tuberculosis prevalence and treatment outcome in an under-resourced setting: The case of Enugu state, South East Nigeria," Nigerian Medical Journal, vol. 54, no. 6, p. 392, 2013.

[12] Punch, "Nigeria ranked 4th in TB infection worldwide - Report," http://www.punchng.com/nigeria-has-4th-tb-infection-rate-worldwide-report/.

[13] "Nigeria Development Data," http://www.demographicdividend.org/country_highlights/nigeria/.

[14] R. Yaesoubi and T. Cohen, "Identifying dynamic tuberculosis case-finding policies for HIV/TB coepidemics," Proceedings of the National Acadamy of Sciences of the United States of America, vol. 110, no. 23, pp. 9457-9462, 2013.

[15] J. V. Lazarus, M. Olsen, L. Ditiu, and S. Matic, “TuberculosisHIV co-infection: Policy and epidemiology in 25 countries in the WHO European region," HIV Medicine, vol. 9, no. 6, pp. 406-414, 2008.

[16] D. Okuonghae and B. O. Ikhimwin, "Dynamics of a mathematical model for tuberculosis with variability in susceptibility and disease progressions due to difference in awareness level," Frontiers in Microbiology, vol. 6, 2016.

[17] T. F. Brewer, G. A. Colditz, S. M. Krumplitsch, S. J. Heymann, M. E. Wilson, and H. V. Fineberg, "Strategies to decrease tuberculosis in US homeless populations: A computer simulation model," Journal of the American Medical Association, vol. 286, no. 7, pp. 834-842, 2001.

[18] P. J. Dodd, R. G. White, E. L. Corbett, and S. P. Tripathy, "Periodic Active Case Finding for TB: When to Look?" PLoS ONE, vol. 6, no. 12, p. e29130, 2011.

[19] P. Kim and C. H. Lee, "Epidemic Spreading in Complex Networks with Resilient Nodes: Applications to FMD," Complexity, vol. 2018, Article ID 5024327, 9 pages, 2018.

[20] A. Corberán-Vallet, F. J. Santonja, M. Jornet-Sanz, and R.-J. Villanueva, "Modeling Chickenpox Dynamics with a Discrete Time Bayesian Stochastic Compartmental Model," Complexity, vol. 2018, Article ID 3060368, 9 pages, 2018.

[21] S. I. Bala and N. M. Ahmad, "Bistability analysis in mathematical model of M-phase control in Xenopus oocyte extracts," Computational and Applied Mathematics, vol. 37, no. 3, pp. 2667-2692, 2018.

[22] C. Ozcaglar, A. Shabbeer, S. L. Vandenberg, B. Yener, and K. P. Bennett, "Epidemiological models of Mycobacterium tuberculosis complex infections," Mathematical Biosciences, vol. 236, no. 2, pp. 77-96, 2012.

[23] M. R. Nyendak, B. Park, M. D. Null et al., "Mycobacterium tuberculosis Specific CD8+ T Cells Rapidly Decline with Antituberculosis Treatment," PLoS ONE, vol. 8, no. 12, p. e81564, 2013.

[24] H. J. Na, J. S. Eom, G. Lee et al., "Exposure to Mycobacterium tuberculosis during flexible bronchoscopy in patients with unexpected pulmonary tuberculosis," PLoS ONE, vol. 11, no. 5, 2016.

[25] L. Telisinghe, K. L. Fielding, J. L. Malden et al., "High tuberculosis prevalence in a South African prison: The need for routine tuberculosis screening," PLoS ONE, vol. 9, no. 1, Article ID e87262, 2014.

[26] P. Nahid, E. E. Bliven, E. Y. Kim et al., "Influence of M. tuberculosis lineage variability within a clinical trial for pulmonary tuberculosis," PLoS ONE, vol. 5, no. 5, 2010.

[27] A. Parhar, Z. Gao, C. Heffernan, R. Ahmed, M. L. Egedahl, and R. Long, "Is early tuberculosis death associated with increased tuberculosis transmission?” PLoS ONE, vol. 10, no. 1, 2015.

[28] J.-M. García-García, R. Blanquer, T. Rodrigo et al., "Social, clinical and microbiological differential characteristics of tuberculosis among immigrants in Spain," PLoS ONE, vol. 6, no. 1, 2011.

[29] J. Millet, A. Orcau, C. Rius et al., "Predictors of Death among Patients Who Completed Tuberculosis Treatment: A Population-Based Cohort Study," PLoS ONE, vol. 6, no. 9, p. e25315, 2011.

[30] Shaip Krasniqi, Arianit Jakupi, Armond Daci et al., "Tuberculosis Treatment Adherence of Patients in Kosovo," Tuberculosis Research and Treatment, vol. 2017, Article ID 4850324, 8 pages, 2017.

[31] S. W. Asgedom, M. Teweldemedhin, and H. Gebreyesus, "Prevalence of Multidrug-Resistant Tuberculosis and Associated Factors in Ethiopia: A Systematic Review," Journal of Pathogens, vol. 2018, Article ID 7104921, 8 pages, 2018.

[32] L. Liu and Y. Wang, "A mathematical study of a TB model with treatment interruptions and two latent periods," Computational and Mathematical Methods in Medicine, vol. 2014, Article ID 932186, 15 pages, 2014.

[33] S. Liu, A. Li, X. Feng, X. Zhang, and K. Wang, "A Dynamic Model of Human and Livestock Tuberculosis Spread and Control in Urumqi, Xinjiang, China," Computational and Mathematical Methods in Medicine, vol. 2016, 2016.

[34] F. A. Krsulovic, M. Lima, and P. Cardona, "Tuberculosis Epidemiology at the Country Scale: Self-Limiting Process and the HIV Effects," PLoS ONE, vol. 11, no. 4, p. e0153710, 2016.

[35] World Health Organization, "Media Centre: Tuberculosis, Fact sheet," 2016.

[36] R. S. Wallis, "Mathematical Models of Tuberculosis Reactivation and Relapse," Frontiers in Microbiology, vol. 7, 2016.

[37] D. P. Moualeu-Ngangue, S. Röblitz, R. Ehrig, and P. Deuflhard, "Parameter identification in a tuberculosis model for Cameroon," PLoS ONE, vol. 10, no. 4, 2015.

[38] S. Ohno, Evolution by Gene Duplication, Springer Berlin Heidelberg, 1970.

[39] B. Song, C. Castillo-Chavez, and J. P. Aparicio, “Tuberculosis models with fast and slow dynamics: the role of close and casual contacts," Mathematical Biosciences, vol. 180, no. 1-2, pp. 187205, 2002. 
[40] G. Guzzetta, M. Ajelli, Z. Yang, S. Merler, C. Furlanello, and D. Kirschner, "Modeling socio-demography to capture tuberculosis transmission dynamics in a low burden setting," Journal of Theoretical Biology, vol. 289, no. 1, pp. 197-205, 2011.

[41] B. M. Murphy, B. H. Singer, and D. Kirschner, "On treatment of tuberculosis in heterogeneous populations," Journal of Theoretical Biology, vol. 223, no. 4, pp. 391-404, 2003.

[42] "Nigeria Tuberculosis Fact Sheet, United States Embassy in Nigeria," 2012.

[43] A. Zumla, A. George, V. Sharma, R. H. N. Herbert, A. Oxley, and M. Oliver, "The WHO 2014 global tuberculosis report-further to go," The Lancet Global Health, vol. 3, no. 1, pp. e10-e12, 2015.

[44] H. H. Kyu, E. R. Maddison, N. J. Henry et al., "The global burden of tuberculosis: results from the Global Burden of Disease Study 2015," in The Lancet Infectious Diseases, vol. 18, pp. 261-284, 2018.

[45] C. Lienhardt, P. Glaziou, M. Uplekar, K. Lönnroth, H. Getahun, and M. Raviglione, "Global tuberculosis control: Lessons learnt and future prospects," Nature Reviews Microbiology, vol. 10, no. 6, pp. 407-416, 2012.

[46] "Centers for Disease Control and Prevention (US) and National Center for Prevention Services (US). Division of Tuberculosis Elimination and National Center for HIV and STD and TB Prevention (US). Division of Tuberculosis Elimination, Reported tuberculosis in the United States; US Department of Health and Human Services, Public Health Service, Centers for Disease Control and Prevention, National Center for Prevention Services, Division of Tuberculosis Elimination," 1999.

[47] USAID, "Health fact sheet," https://www.usaid.gov/sites/ default/files/documents//USAID-Nigeria-Health-Fact-Sheet .pdf.

[48] Vanguard News paper, "Nigeria overtakes India as world's poverty capital - Report," https://www.vanguardngr.com//06/ nigeria-overtakes-india-as-worlds-poverty-capital-report/ Vanguard News paper.

[49] A. Zwerling, S. Shrestha, and D. W. Dowdy, "Mathematical Modelling and Tuberculosis: Advances in Diagnostics and Novel Therapies," Advances in Medicine, vol. 2015, Article ID 907267, 10 pages, 2015.

[50] N. A. Menzies, T. Cohen, H.-H. Lin, M. Murray, and J. A. Salomon, "Population health impact and cost-effectiveness of tuberculosis diagnosis with Xpert MTB/RIF: a dynamic simulation and economic evaluation," PLoS Medicine, vol. 9, no. 11, Article ID e1001347, 2012.

[51] C. J. L. Murray and J. A. Salomon, "Modeling the impact of global tuberculosis control strategies," Proceedings of the National Acadamy of Sciences of the United States of America, vol. 95, no. 23, pp. 13881-13886, 1998.

[52] "Nigeria Tuberculosis profile," http://www.who.int/tb/country/ data/profiles/en/profile.

[53] D. R. Nagpaul, M. K. Vishwanath, and G. Dwarakanath, "A socio-epidemiological study of out-patients attending a city tuberculosis clinic in India to judge the place of specialized centres in a tuberculosis control programme.," Bulletin of the World Health Organization, vol. 43, no. 1, pp. 17-34, 1970.

[54] D. Banerji and S. Andersen, "A sociological study of awareness of symptoms among persons with pulmonary tuberculosis," Bulletin of the World Health Organization, vol. 29, pp. 665-683, 1963.

[55] A. J. Rubel and L. C. Garro, "Social and cultural factors in the successful control of tuberculosis," Public Health Reports, vol. 107, no. 6, pp. 626-635, 1992.
[56] World Health Organization, “The End TB Strategy," http://www .who.int/tb/End_TB_brochure.pdfStrategy.

[57] M. W. Rosegrant, M. C. Agcaoili-Sombilla, and N. D. Perez, Global Food Projections to 2020: Implications for Investment, Diane Publishing, 1995. 


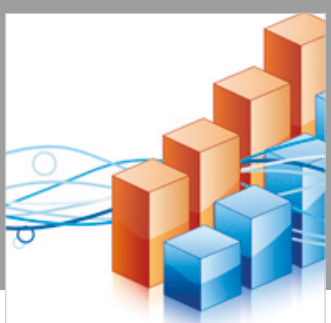

Advances in

Operations Research

\section{-n-m}
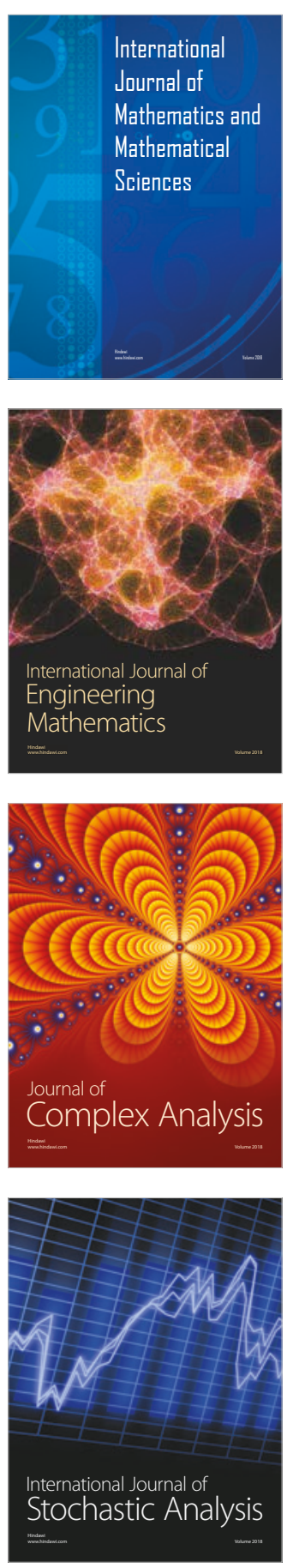
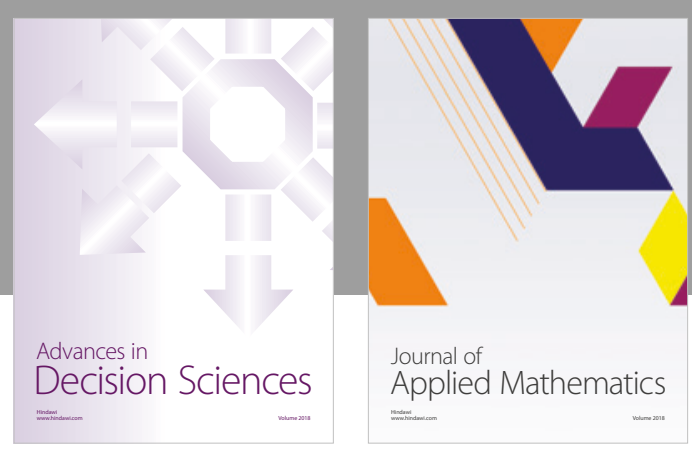

Journal of

Applied Mathematics
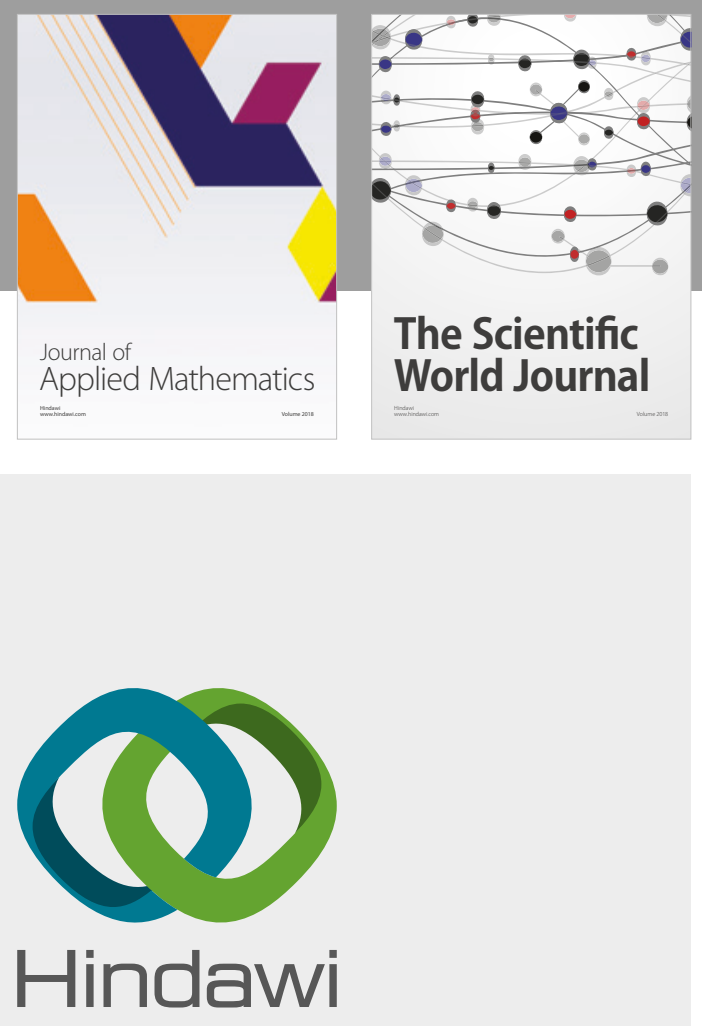

Submit your manuscripts at

www.hindawi.com

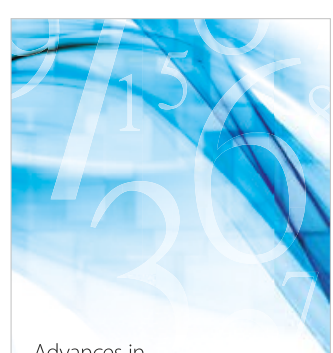

Advances in
Numerical Analysis
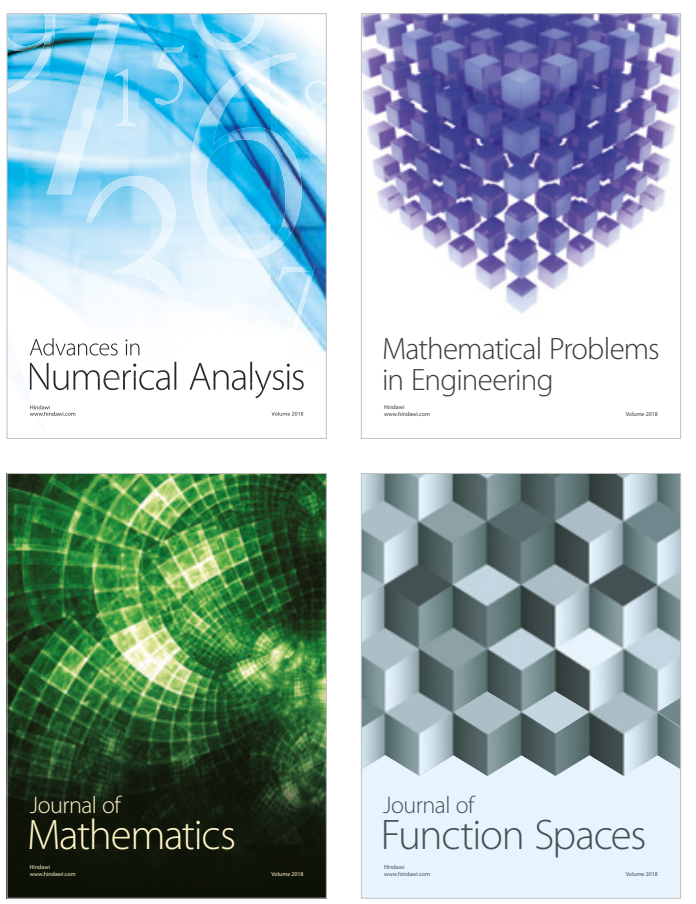

Mathematical Problems in Engineering

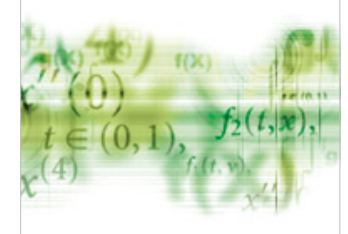

International Journal of

Differential Equations

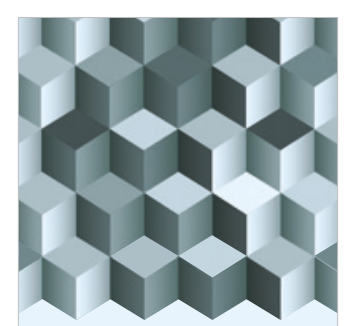

Journal of

Function Spaces
The Scientific

World Journal

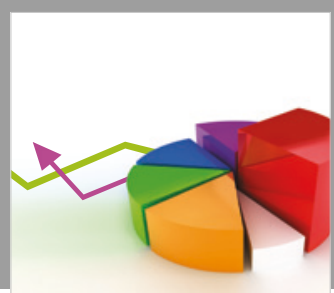

Journal of

Probability and Statistics
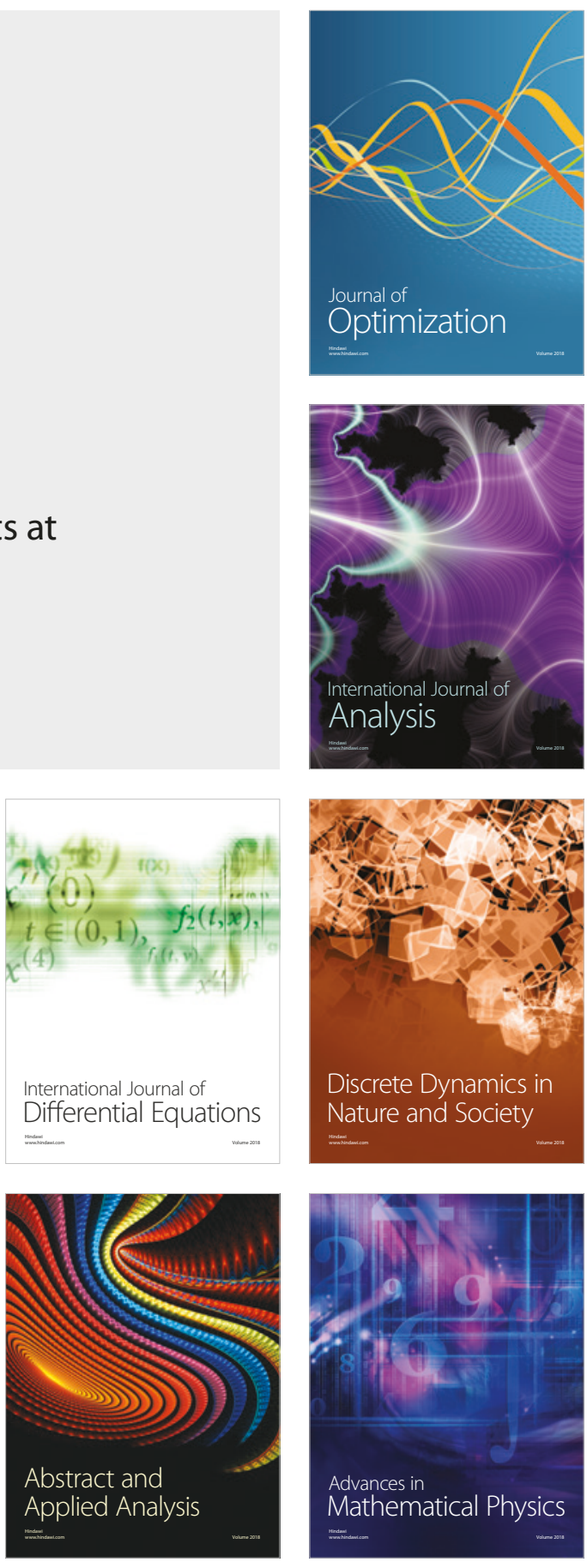